\title{
3D Pain Drawings and Seating Pressure Maps: Relationships and Challenges
}

\author{
Fotios Spyridonis and Gheorghita Ghinea \\ School of Information Systems, Computing and Mathematics \\ Brunel University \\ Uxbridge, UK \\ Email \{Fotios.Spyridonis, George.Ghinea\}@brunel.ac.uk
}

\begin{abstract}
Mobility impaired people constitute a significant portion of the adult population, which often experience back pain at some point during their lifetime. Such pain is usually characterized by severe implications reflected on both their personal lives, as well as on a country's health and economic systems. The traditional 2-dimensional (2D) representations of the human body often used can be limited in their ability to efficiently visualize such pain for diagnosis purposes. Yet, patients have been shown to prefer such drawings. However, considering that pain is a feeling or emotion that is subjective in nature, the pain drawings could be consequently regarded as a subjective means of communicating such pain. As a result, the work described in this paper proposes an alternative, which encompasses a 3dimensional (3D) pain visualization solution, developed in a previous work of ours. This alternative is complemented with the upcoming technique of pressure mapping for more objectivity in the pain data collection. The results of this study have shown that the proposed approach is a promising solution for the purpose intended, and it could generally prove to be a significant complementary method in the area of medical practice for the mobility impaired community.
\end{abstract}

Index Terms-Pain Drawings, 3D Visualization, Pain Visualization, Mobile Data Collection, Pressure Mapping, Objective Pain Measurements

\section{INTRODUCTION}

Evidence arising from past studies reveals that Low Back Disorders (LBDs) represent an uncomfortable experience that appears to predominantly affect most of the western and industrialized societies. Specifically, the lifetime prevalence of back pain is more than $70 \%$ in most industrialized countries [3], with estimations showing that it affects between $60 \%$ and $90 \%$ of the general population. Research has revealed that four in five adults experience back pain [1] sometime in their lives, usually beginning between ages of the 30 and 40 years [13].

Coming only second to the common cold, LBDs constitute one of the most frequent reasons to seek medical advice, a situation that accounts for tremendous costs to a country's National Health Service (NHS). Specifically, 2008 figures indicate that the NHS in the UK spends more than $£ 1$ billion on back pain related costs per year [1].

\section{A. Back Pain and Mobility Impaired People}

Amongst the significant percentage of the population suffering from some form of LBDs, it seems that there is a trend for back pain to be more common for mobility impaired people. This group of individuals usually find themselves suffering from particularly severe back pain, often deteriorated due to their reliance on wheelchair support that presupposes prolonged sitting and reduced physical activity. In a study carried out by [6], 26\% of electric-powered indoor or outdoor wheelchair (EPIOC) users in the UK admit to pain or discomfort when sitting in their chair at four months after delivery. This figure rises to $46 \%$ at two years, an indication of increasing pain due to prolonged sitting. Similarly, results of another study indicate that back pain was a common problem in the studied group with a reported frequency of always (12\%), everyday (33\%), several times a week (17\%), sometimes (30\%), and very seldom (8\%) [24].

However, despite the huge amount of money and resources spent, the assessment of this medical complaint remains notoriously difficult, with sporadic success in diagnosing back pain. The reason for this lies in the fact that the various assessment tools normally used are limited in their ability to adequately visualize and communicate such pain to clinicians. To this end, the focus of the work described in this paper has been to overcome the aforementioned limitations with the augmentation of such assessment tools with a novel, prototype method - in the anticipation that it can make an important contribution to the back pain data collection and monitoring process, as well as support the reduction of healthcare costs.

\section{BACK PAIN MONITORING}

The diagnosis and treatment of LBDs and specifically of back pain is a major health problem whose assessment remains notoriously difficult. This challenge typically stems from the multidimensional nature of pain, which typically involves physiologic, as well as emotional qualities [17]. As a result of its heterogeneity, the available medical information only provides partial success in the diagnosis and treatment of this chronic disease, with only $15 \%$ of the patients obtaining an accurate diagnosis [30].

Specifically, considering the above, there are two important factors according to the literature, which could affect this partial success in diagnosis, namely:

a) The limited visualization ability of the current assessment tools, and

b) The insufficient communication of pain to clinicians 


\section{A. Back-pain Visualization}

According to the literature, several tools that attempt to address the nature of back pain exist and are typically in the form of a questionnaire. The usefulness of these tools is mainly twofold - firstly, by using them, comprehensive information related to a patient's medical history could be acquired. Secondly, specific clinical information regarding the basic understanding of pain description, intensity, and pain location could be similarly collected [15].

Specifically, three tools have been traditionally used to measure pain intensity, namely Visual Analogue Scale (VAS) / Graphic Rating Scale (GRS), Verbal Rating Scale (VRS), and Numerical Rating Scale (NRS) [14]. However, for the purpose of the work presented in this paper, only the clinical information tools related to the ability to more efficiently visualize back pain is of considerable interest. To this end, the most important tool currently in use to indicate the location of pain is a diagram, usually a two-dimensional representation of a human body, on which the patient is asked to mark where the pain is located, and the type of pain that he or she is suffering from. This type of diagram is known in the literature as a "pain drawing" and is shown in Fig. 1a.

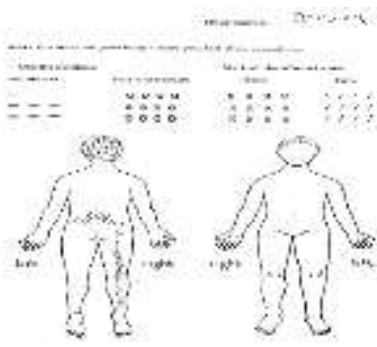

Fig. 1a 2D Pain Drawing

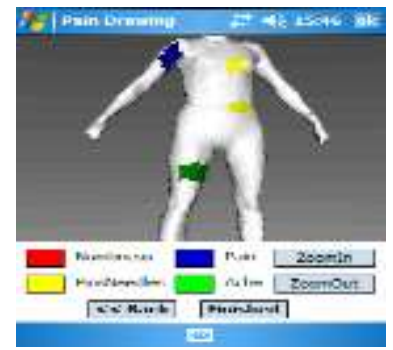

Fig. 1b 3D Pain Drawing
The consensus of the literature seems to indicate that the pain drawing is considered to be a valuable and useful tool in identifying pain location and sensation type, with most of the studies pointing to patients consistently completing it $[21,28]$. Nevertheless, based on a previous paper of ours [5], the aforementioned pain drawing was found to be subject to several drawbacks, especially regarding its limited ability to accurately visualize the medical information required in all spatial dimensions. Therefore, an enhanced, 3D version of the pain drawing was implemented (Fig. 1b), with the ability to more accurately visualize back pain-related information [5].

\section{1) Evaluation of the 3D Pain Drawing}

The 3D pain drawing developed was consequently evaluated, with the purpose of this evaluation being to identify the opinions of the most important stakeholders that would eventually use it. Thus, initially four clinicians from two different London hospitals, with significant experience in 2D pain drawings, were approached and asked to review the 3D pain drawing. In general, all clinicians surveyed approved of the visual appearance and usability of the enhanced pain drawing, and further suggested that it would be usable in a clinical environment.

In a similar manner, a second evaluation was carried out with respect to the necessary patient opinions as the direct users of the diagram. Specifically, 45 patients (26 males, 19 females, mean age 46.1 years) have evaluated the 3D pain drawing, all voluntarily recruited from Northwick Park Hospital, and the U.K. National Forum of Wheelchair User Group. In agreement with the clinician's opinions, the results have shown that with respect to the developed 3D interface, the enhanced pain drawing was perceived very positively, and with great enthusiasm for the purpose intended. More information regarding the evaluation can also be found in [5].

\section{B. Communication of Pain}

Although the pain drawing as a tool is widely considered to be valid [5, 21, 23], there seems to be considerable debate as to whether assessments of pain drawings alone are sufficient to communicate the pain to a clinician, and, indeed, of determining whether someone really suffers or not from pain $[7,9,14,17,20,21,25]$.

Typically, patient self-reporting is the most reliable indicator of the existence and intensity of pain [11]. However, self-reporting is subjective by definition, since patients that self-report back pain may have developed psychological or emotional problems due to the fact that they have to deal with such pain. This view is also supported by [18] definition in which "pain is whatever the experiencing person says it is and exists whenever they say it does", as well as by [10] who support that "pain is a personal and subjective experience that can only be felt by the sufferer". Based on the above, the various pain measurement tools used for back pain assessment are also considered to be subjective in nature, since they are the direct indicators of a patient's self-reporting of back pain. Moreover, [9] also found similar results in their study about the use of pain drawings in identifying real or imagined pain. Specifically, the results suggest that subjective assessments of pain drawings alone are not sufficient in determining whether someone really suffers or not from pain.

To this end, the need for the establishment of a reliable objective back pain measurement method has been identified, which would ideally complement the aforementioned subjective measurements in more accurately assessing the patients' subjective back pain indications as communicated through a pain drawing. To the best of our knowledge, to date there are no accurate and reliable methods that can be used to objectively and effectively measure back pain, an opinion also supported by [11].

\section{Objective Pain Measurements}

The clinical literature contains a variety of methods which have been extensively described and exploited in order to acquire such objective measurements of pain [8], with these being mainly related and limited to physical body functionality measurements. To this end, several physiological (e.g. Range of Motion, Shoulder tests, etc.) and neurological (e.g. Muscle Strength, Nerve Stretch, etc.) examination tests that address the aforementioned aspects are usually considered for objective pain measurements [2,16]. Unfortunately, according to [8], such objective measures often tend to be less reliable even compared to patient-reported subjective measures (e.g. VAS and pain drawings), usually because of the examiner's lack of ability to "reliably quantify patient function".

Indeed, the issue of reliability of such traditional objective 
measures applied to back pain seems to be a recurring theme in the literature [11]. Although physical examinations, laboratory tests, and imaging techniques are considered objective measurements, in reality they are also influenced by the patient's motivation, effort, and psychological state [19]. What could be done to improve the situation, thus, is to identify an alternative objective assessment method that would ideally minimize the aforementioned risks.

Consequently, several studies have exploited pressure mapping - a means of assessing the pressure distribution between a person's thighs and buttocks and the seating surface [26], as an assessment tool on patients with various conditions that involve some form of pain. The results of the study reported in [26] have shown the usefulness of visual pressure maps, especially with wheelchair users. Accordingly, [29] used pressure measurements and mapping on patients with Spinal Cord Injuries (SCI), with a view to reducing pain originating from pressure sores developed due to inappropriate wheelchair cushions and unsuitable posture. Similarly, [4] examined the use of pressure measurements also related to wheelchair cushioning, with again very promising results for the use of pressure mapping on the elderly population regarding reducing pain coming from pressure ulcers.

Considering that it does not rely on patient subjective selfreports, the usefulness of pressure mapping as an objective communication tool for characterizing pain is well established. However, to the best of our knowledge no study currently exists in the literature exploring the relationship between objective pressure maps and subjective experiences of pain in a wheelchair population. Specifically, no study has ever combined the use of pain drawings and pressure mapping's ability to communicate pain more efficiently, for the purpose of assessing back pain patients in the wheelchair population.

\section{METHODS}

\section{A. Overview of the Methodology}

The aim of this study is to examine the relationship between subjective and objective back pain measurements. To this end, we specifically targeted two research objectives: a) to examine relations that exist between a $3 \mathrm{D}$ pain drawing and its corresponding pressure maps, and $b$ ) to measure the efficiency of the 3D pain drawing in visualizing back pain, in respect of its corresponding pressure maps.

For the former, a visual interpretation of the acquired information will be attempted, backed up by analysis of the numerical data produced by the pressure mapping equipment. For the latter, it has to be initially noted that we define efficiency in terms of the surface area indicated by both methods. We also note that according to ISO-9241[22], efficiency metrics include the number of clicks required to accurately complete a certain task. Accordingly, in terms of the 3D pain drawing's usability, fewer attempts ('clicks' on the drawing's body surface) to indicate the pain location is better, since each attempt corresponds to roughly the same amount of surface area being selected. Thus, what we would like to identify is which of the two methods captures more 'tightly' (with least amount of surface area indicated) pain data.

\section{B. Instrumentation}

The instrumentation used for this study consists of a HP iPAQ hx2400 PDA running the Microsoft Windows Mobile 5.0 operating system, on an Intel $520 \mathrm{Mhz}$ PXA270 processor containing $64 \mathrm{MB}$ standard memory, as well as $128 \mathrm{MB}$ internal flash ROM [5]. Moreover, a laptop running Microsoft Windows Vista that records the information collected through the commercially available FSA (VistaMed, Canada) pressure mapping device, was also used. The pressure mapping device consists of a sensor mat (16x16 array of sensors), a computer interface module, and software that runs on the laptop to record the information from the pressure sensors. The sensor mat was calibrated prior to the beginning of data collection according to the manufacturer's recommended procedure. Lastly, no specific cushioning or type of wheelchairs was used, as the aim was to identify the relationship between the subjective and objective measures, and not to propose or evaluate any appropriate cushioning or wheelchair.

\section{Description of Subject Group}

Nine subjects ( 3 female; 6 male, mean age 62.6 years, range 43-82) volunteered to participate in the research study. All were recruited from the Hillingdon Independent Wheelchair User Group, London, UK and from the London Borough of Hillingdon council. Their diagnoses varied and included one or more of the following: Cerebral Palsy (CP), Multiple Sclerosis (MS), severe back or hip pain, and arthritis. The criteria for selection was that the subject be a wheelchair user with an age of 20 years or greater and have pain only in the lower body parts. From the nine subjects, seven were found to be eligible to participate ( 3 female; 4 male), as two of them did not meet the selection criteria. For illustrative purposes and due to space constraints, only the four out of the seven participants will be discussed in this paper. Subsequently, the final mean age was finally calculated as 62.4 years, with a range of 43-82 years. Finally, the mean pain intensity was 5.28 on a Visual Analogue Scale (0-no pain; 9-worst pain), with all participants having experienced pain for over a year.

\section{Protocol and Algorithm}

Prior to initiation of measurements, informed consent was obtained by each participant along with general and clinical information. Clinical information consisted of their diagnosis, disabilities and medical conditions, factors that worsen/relief their pain, medication received, and pain intensity.

Subjective and objective measurements were taken in parallel, and started with each participant in turn being asked to take a position on the pressure mat and make sure that they adjust their posture to their most comfortable sitting position. Once done, an initial pressure measurement was taken to record the pressure when sat for the first time on the chair. To be more specific, a pressure measurement is taken by placing a pressure mat between the patient's buttocks and thighs, and the seating surface. Subsequently, data computed from the sensors is recorded and displayed on the computer screen in various forms, including a color-coded contour map (Fig. 2), a threedimensional grid, and numerical pressure values [27]. 

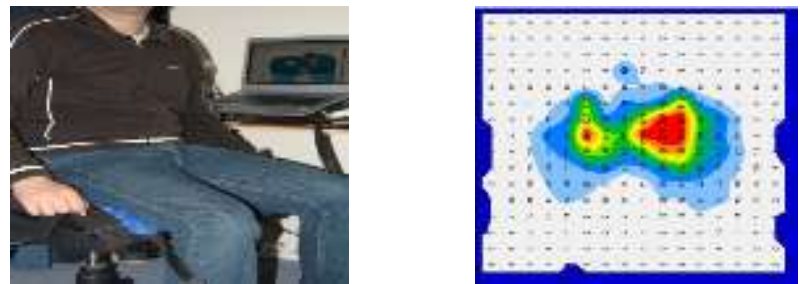

Fig. 2 Pressure Mapping Equipment and Result

After an 8 minute sitting time on the pressure mat, which was identified as the 'optimal settling time prior interface pressure recording' [27], the subject was asked to pinpoint on the 3D pain drawing the location and type of their pain, while at the same time a final pressure measurement was recorded in parallel with the completion of the 3D drawing. The initial and final pressure measurements were both taken to identify how pressure escalates after a sitting period of time, something that could possibly help us understand if subjective measures are linked with the objective measures. Each session had a duration of approximately 25 minutes. After the end of each session, another subject would take a position on the pressure mat, and the protocol was repeated.

\section{E. Data Analysis}

Two sets of data were generated by the measurements: the 3D pain drawings and the FSA pressure maps. Being both graphical data, the analysis of these two sets of data initially consisted of a visual interpretation, and comparison of the 3D pain drawings and pressure maps produced for each of these drawings, in order to examine the relationship between them. It has to be mentioned at this point that the words 'relationship/relation' are not used throughout this paper in the statistical sense, but rather to describe a connection that might exist between the two data sets, as derived from a visual interpretation. The reason lays in the fact that such a topographical representation and interpretation is very useful in summarizing a patient's description of the location and type of pain, in an interpretable way for the clinician. Moreover, it makes it possible to determine whether the pain experienced is of organic or non-organic nature [28].

Additionally, further numerical analysis was also sought, in order to identify whether statistical evidence occurs in support of the graphical results produced. The pressure values produced from the mat's sensors were collected, and this raw data was used to calculate average pressure variations. Finally, a similar numerical analysis on the 3D pain drawing and the pressure values was also generated, in order to measure the efficiency of our proposed method.

\section{RESUltS}

We have identified relations between a patient's 3D pain drawing and the corresponding pressure maps for all seven participants of the study. These relations have been classified as either direct or indirect. Specifically, the former describes the case where the pinpointed pain locations on the 3D pain diagram match with the pressure areas identified on the pressure maps, and therefore, the pain reported could be directly indicated by this pressure. Similarly, the latter describes the case where the pain locations do not match with the pressure areas identified, yet, the pain reported could be indirectly indicated by this pressure. These relations will be demonstrated in the following sections for four out of the seven participants by including snapshots of the 3D pain drawing completed by each participant, as well as of their corresponding recorded pressure maps.

\section{A. Visual Interpretation}

The four subjects, who were diagnosed with lower hip pain, back pain, and arthritis (Table I) were identified as having a relation between their 3D pain drawing and their corresponding pressure maps. Specifically, their subjective and objective measures were either directly or indirectly linked, as can be concluded from a visual interpretation of the data acquired. Fig. 3 shows, for example, a direct relation between the 3D pain drawing and the two pressure maps obtained for the first participant. Similarly, Fig. 4 shows another direct relation between the $3 \mathrm{D}$ pain drawing and the two pressure maps obtained for the second participant.

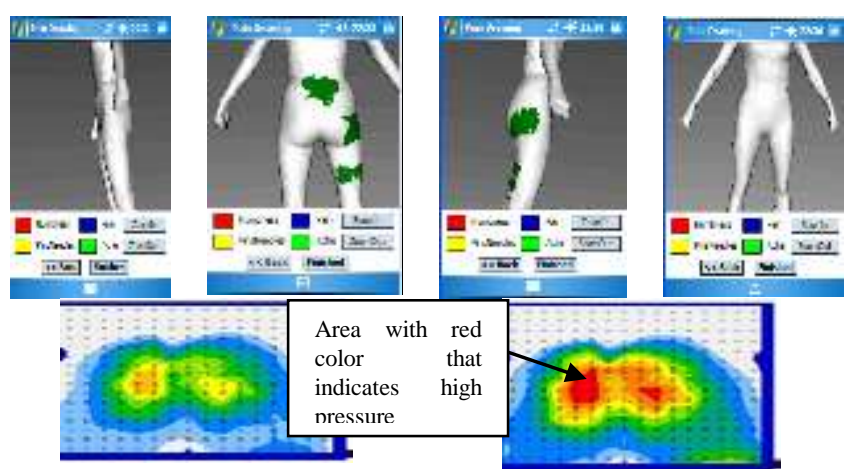

Fig. 3 3D Pain Drawing with Initial (left) and Final (right) Pressure Maps for Participant 1

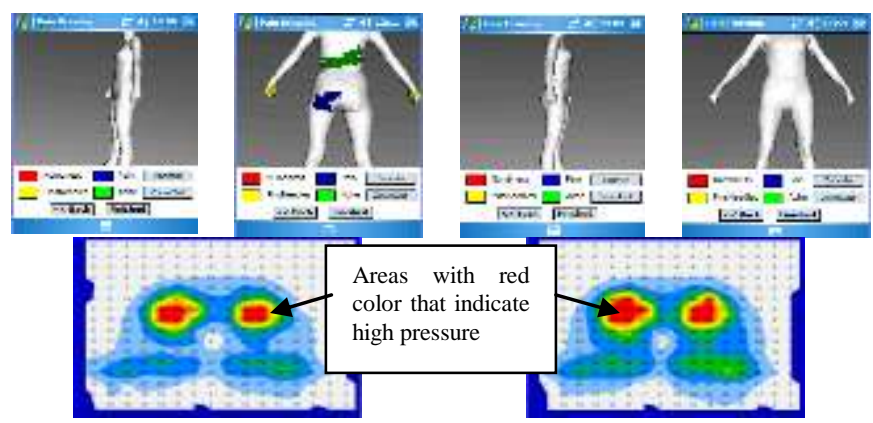

Fig. 4 3D Pain Drawing with Initial (left) and Final (right) Pressure Maps for Participant 2

Specifically, in Fig. 3 we see that in the initial pressure map there was no high pressure when the subject first sat on the chair (high pressure is indicated in the pressure mapping system with the red color). Eight minutes later, he pinpointed on the pain drawing the locations of his pain. At the same time, the final pressure map was recorded. From the 3D pain drawing we can see that he experiences ache in his right leg, buttocks and in his lower back. Accordingly, the final map shows an increase over time in pressure on the right side of the buttocks, visually justifying the ache experienced, which could be directly indicated by the increase in pressure while the subject was seating for prolonged time. 
Analysis of the numerical data acquired directly as recorded from the pressure sensors was also performed for all four participants. The results of this analysis are presented in Table I, where we can see that there is a positive pressure variation between the initial pressure recording and the final pressure recording for the participant with lower back pain, an indication that there is an increase in pressure over time, in support of the visual interpretation provided.

In the same manner, from Fig. 4 it has to be noted that the participant's initial pressure map was indicating high pressure from the moment she first sat on the chair. Eight minutes later, on the 3D pain drawing she reported that she is experiencing pain in her right hip and buttocks, as well as ache in her lower back. By carefully examining the final pressure map, we can identify that the pressure surface of her buttocks increases over time, yet, the high pressure values remain approximately the same as when she first sat. It is safe to conclude that her pain and ache could be directly indicated by the high pressure produced by seating for a prolonged time, as indicated by both maps. However, when compared to the first participant, we cannot safely assume that they are directly indicated by the pressure increase, since according to the final map, the pressure remains essentially the same over time.

From Table I we can see the positive pressure variation for the second participant. This could be an indication of the existence of an increase in pressure over time, something that was not clearly identified by the visual interpretation of the pressure maps alone. Nevertheless, this supports the assumption that pain could be directly indicated by the increased pressure while seating for prolonged time.

Two of the subjects who were respectively diagnosed with lower back pain and arthritis, were found to have an indirect relation between their subjective and objective measures. Fig. 5 shows for example such an indirect relation between the 3D pain drawing and the two pressure maps acquired from the third participant. The initial pressure map indicated very little pressure when the patient first sat on the chair. Moreover, pain shown on the below 3D drawing was mainly in the left hip and knee, as well as in the lower back, however, the final map recorded eight minutes later showed high pressure on the right buttocks area.

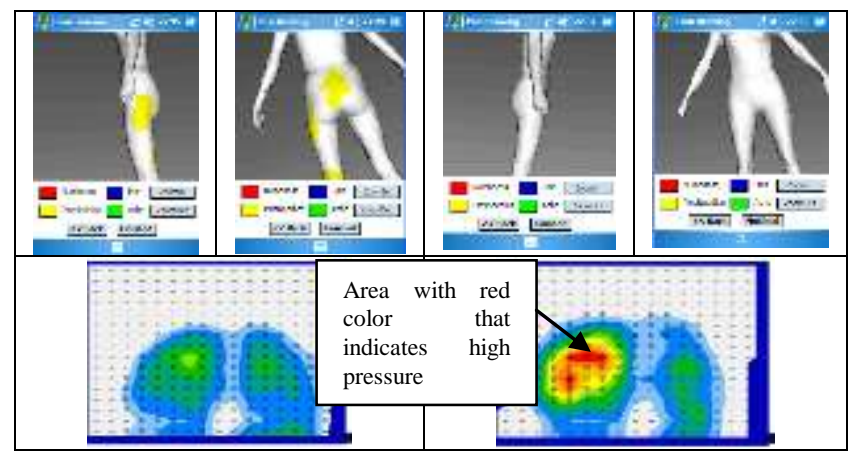

Fig. 5 3D Pain Drawing with Initial (left) and Final (right) Pressure Maps for Participant 3

Considering the above information, an indirect relation between the pain locations on the 3D pain drawing and the corresponding pressure maps seems to exist; thus, in this case increased pressure in the right buttocks over time does not seem to directly visually indicate the pain experienced in the left side. By consulting the subject though, she let us know that because she experiences pain mainly in her left side, she tends to lean to her right for relief, and that explained the high pressure shown on the final map. Therefore, we could assume that the pressure increase over time could be an indirect indication this time of the pain experienced, compared to the previous two cases where pain was proved as been directly indicated by such a pressure increase. Moreover, further investigation of the specific pressure maps shows a striking asymmetry in the indicated pressure, a finding that could possibly further reveal hidden back pain information. In support of the visual interpretation, a positive pressure variation appears for this participant as well (Table I), which also demonstrates the increase in pressure over time. Similarly, Fig. 6 shows the indirect relation between the 3D pain drawing and the pressure maps obtained for the fourth participant.

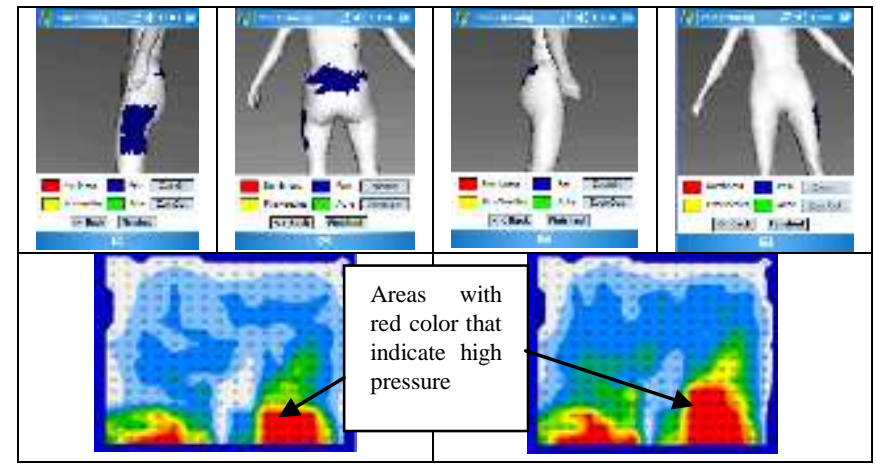

Fig. 6 3D Pain Drawing with Initial (left) and Final (right) Pressure Maps for Participant 4

Again, by observing the above figures we don't clearly see how the pain locations on the 3D pain drawing directly match with the pressure maps, since high pressure is mainly indicated in both the participant's thighs. Although pain reported on the pain drawing in the left thigh could be indicated by the high pressure on the left side on both pressure maps, no signs of pressure exist in the buttocks. This could be an indication of lower back pain, which was also reported by the participant. However, along the same lines of the previously mentioned case, certain conclusions could be reached from the pressure maps regarding this person's posture and sitting habits, which could in turn lead to identify the possible causes of her pain.

TABLE I

NUMERICAL ANALYSIS OF PRESSURE SENSOR DATA

\begin{tabular}{|c|c|c|c|c|c|}
\hline $\begin{array}{c}\text { Participant } \\
\text { Number }\end{array}$ & $\begin{array}{c}\text { Participant } \\
\text { Diagnosis }\end{array}$ & $\begin{array}{c}\text { Initial } \\
\text { Total } \\
\text { Pressure } \\
(\mathbf{m m H g})\end{array}$ & $\begin{array}{c}\text { Final } \\
\text { Total } \\
\text { Pressure } \\
(\mathbf{m m H g})\end{array}$ & $\begin{array}{c}\text { Pressure } \\
\text { Variation } \\
(\mathbf{m m H g})\end{array}$ & $\begin{array}{c}\text { Average } \\
\text { Pressure } \\
\text { Variation } \\
\text { per } \\
\text { Sensor } \\
(\mathbf{m m H g})\end{array}$ \\
\hline 1 & $\begin{array}{c}\text { Lower back } \\
\text { pain }\end{array}$ & 10906.44 & 16039.02 & 5165.86 & 26.356 \\
\hline 2 & $\begin{array}{c}\text { Severe hip } \\
\text { pain }\end{array}$ & 7436.05 & 8819.93 & 1451.68 & 7.976 \\
\hline 3 & Arthritis & 6477.18 & 8663 & 3978.54 & 25.341 \\
\hline 4 & Back pain & 12825.21 & 16537.33 & 3792.8 & 17.084 \\
\hline
\end{tabular}




\section{CONCLUDING DISCUSSION}

Specifically, the participant seems to be leaning forward in her attempts to relieve her pain. This causes high pressure over time, which could indirectly indicate the lower back pain expressed, due to the bad posture taken while seating for prolonged periods of time. As before, there is a positive pressure variation (Table I), which also demonstrates this increase in pressure over time, in support of the visual interpretation provided.

\section{B. Back-pain Visualization Efficiency}

Considering that relationships do exist between the 3D pain drawing and the pressure maps, as previously discussed, it is also worthwhile to investigate how efficient (in terms of occupying the least surface area) the $3 \mathrm{D}$ pain drawing is in visualizing back pain in the context of these relationships. To this end, a comparison between the percentage of the body surface area selected on the $3 \mathrm{D}$ pain drawing to indicate the pain location, and the corresponding percentage of the surface area indicated on the pressure map, was performed to examine which of the two methods better captures pain data more efficiently (Table II).

TABLE II

PERCENTAGE OF SURFACE AREA INDICATED IN BOTH METHODS

\begin{tabular}{|c|c|c|c|}
\hline $\begin{array}{c}\text { Participant } \\
\text { Number }\end{array}$ & $\begin{array}{c}\text { Participant } \\
\text { Diagnosis }\end{array}$ & $\begin{array}{c}\text { 3D Pain Drawing } \\
\text { Surface Area } \\
\text { Selected (\%) }\end{array}$ & $\begin{array}{c}\text { Pressure Map } \\
\text { Surface Area } \\
\text { Indicated (\%) }\end{array}$ \\
\hline 1 & Lower back pain & 13.75 & 56.25 \\
\hline 2 & Severe hip pain & 17.5 & 25.39 \\
\hline 3 & Arthritis & 15 & 26.56 \\
\hline 4 & Back pain & 11.25 & 53.90 \\
\hline
\end{tabular}

For the 3D pain drawing case, the body of the mannequin was segmented into clinically appropriate regions after clinical consultations [5]. The percentage of the surface area was measured based on the number of the selected surface regions indicated by the participant, out of the total number of the regions into which the back side of the 3D human mannequin body was divided. It has to be noted that we only consider the body regions from the lower back to the knees, as this is where back pain normally occurs [12].

Given that a pressure map is made up of a matrix of $16 \times 16$ sensors, a cell associated with a particular sensor is deemed to indicate an area of high pressure if the reading of their sensor is above $48.88 \mathrm{mmHg}$, a figure which represents the average final pressure per sensor for all four cases discussed in this paper. Accordingly, the second column of Table II expresses, in percentage terms, the fraction of such sensors (indicating higher than average pressure) out of the total 256 of the mat.

Therefore, considering the above table, the results produced indicate that in overall, the $3 \mathrm{D}$ pain drawing seems to more efficiently localize pain data as compared to the pressure mapping equipment used. Specifically, the amount of the surface area selected for the former is significantly less than the equivalent amount indicated by the latter, a result that supports our second research objective. Nevertheless, the aforementioned discussion aimed to only examine the efficiency of the 3D pain drawing method, yet, for the purpose of back pain assessment both suggested methods should be used complementarily.
The purpose of our current work is twofold: firstly, to determine whether relationships between the 3D pain drawing and the pressure maps exist; secondly, to explore which of the two methods is more efficient at providing pressure/pain maps. Although both aforementioned methods seem to be clinically useful when used in isolation, they have never been tested together to evaluate if both of these ways of measurement are related.

To this end, with regards to our first research objective, the current study has revealed mixed results: whilst for two of the study's participants a direct link was found between the two methods, for the other two participants this relationship was indirect. These results show that high pressure might not necessarily be a possible direct indication of pain, but, could reveal further information pointing to its existence.

Although various studies have been conducted in the literature $[4,26,29]$ that also exploited pressure mapping in the mobility impaired population, their main trend was to reduce any pain originating from pressure sores by suggesting either more appropriate wheelchair cushions, or more suitable postures with respect to the results produced. In contrast, the innovation in our work lies in the fact that, as compared with the aforementioned studies, the intended purpose is to identify such pain in order to prevent its consequences, rather than reducing it as a result of them. To this end, the study presented in this paper has produced very promising results, especially regarding the ability of complimentarily using both of the proposed methods to better and more efficiently indicate back pain, as supported by the relationships identified.

The aforementioned efficiency was justified as part of our second research objective, by measuring the ability of the 3D pain drawing to better localize pain, using the least possible number of clicks to indicate its location on the body surface. To the best of our knowledge, no study currently attempts to evaluate the efficiency of a pain drawing in localizing pain in terms of surface area. Considering the enhanced ability of our proposed 3D pain drawing to better visualize pain [5], and with regards to this study's results that have revealed its usefulness in more efficiently localizing pain, it could be surmised that it is a promising initiative in the clinical literature for the purpose intended.

Nevertheless, it has to be also made clear that this work is prototypical. Therefore, it has not yet been tested in a clinical setting. In addition, we are aware that the subject group is considerably small, and this does not allow us to make any large scale generalizations. However, the pain patterns that exist when combining our proposed methods are obvious even to non-clinicians, which makes it even more imperative that they are also investigated from a clinical point of view.

This paper has thus raised attractive future directions, chief of which is the testing of our methodology in a clinical setting, where more results coming from a more experienced eye could be produced. Moreover, possibly in clinical future work and studies our proposed alternative could be considered as a valuable complementary method. Finally, it would also be worthwhile investigating the asymmetric pain patterns identified in Fig. 5, as they could reveal information valuable 
to the pain community. All these efforts constitute an essential part of our future endeavors.

\section{REFERENCES}

[1] Backcare (2008) Available at:http://www.backcare.org.uk/335/Facts\%20and\%20figures.html [Accessed: 15th October 2009].

[2] B. Bertilson, M. Grunnesjö,, S. Johansson, and L. Strender, "Pain Drawing in the Assessment of Neurogenic Pain and Dysfunction in the Neck/Shoulder Region: Inter-Examiner Reliability and Concordance with Clinical Examination”. Pain Medicine, 8 (2), 134-146, 2007.

[3] D.G. Borenstein, "A clinician's approach to acute low back pain". American Journal of Medicine, 102(1A),16S-22S, 1997.

[4] D.M. Brienza, P.A. Karg, and C.E. Brubaker, "Seat Cushion Design for Elderly Wheelchair Users Based on Minimization of Soft Tissue Deformation Using Stiffness and Pressure Measurements," IEEE Transactions on Rehabilitation Engineering, 4 (4), 320-327, 1996.

[5] G. Ghinea, F. Spyridonis, T. Serif, and A.O. Frank, "3-D Pain Drawings-Mobile Data Collection Using a PDA," IEEE Transactions on Information Technology in Biomedicine, 12 (1), 27-33, 2008.

[6] J. Gibson, and A. Frank, "Pain experienced by electric-powered chair users: a pilot exploration using pain drawings". Physiotherapy Research International, 10 (2), 110-115, 2005.

[7] M. Haefeli, and A. Elfering, "Pain assessment". European Spine Journal, 17-24, 2006

[8] B.T. Harcourt, M. Wijesinha, and G.E. Harcourt, "Subjective and Objective Numerical Outcome Measure Assessment (SONOMA). A Combined Outcome Measure Tool: Findings on a Study of Reliability". National University of Health Sciences, 481-492, 2003.

[9] R.N. Jamison, G.J. Fanciullo, and J.C. Baird, "Usefulness of Pain Drawings in Identifying Real or Imagined Pain: Accuracy of Pain Professionals, Non-professionals, and a Decision Model". The Journal of Pain, 5 (9), 476-482, 2004.

[10] J. Katz, and R. Melzack, "Measurement of pain" Surgical Clinics of North America, 79, 231-252, 1999.

[11] H. Kendall, J. Spence, and M.F. Murphy, "Review of PainMeasurement Tools". Annals of Emergency Medicine, 27 (4), 427-432, 1996.

[12] Kinkade, S. "Evaluation and Treatment of Acute Low Back Pain", American Family Physician, 75 (8), 1181-1188, 2007.

[13] F.C. Koelink, "Chronic Back Pain" Can. Fam. Physician, 36, $1173-$ 1177, 1990.

[14] S.J. Lee, "Pain measurement: Understanding existing tools and their application in the emergency department". Emergency Medicine, 279287,2001
[15] L. Lin, P.J. Hu, and O.R. Sheng, "A decision support system for lower back pain diagnosis: Uncertainty management and clinical evaluations". Science Direct, 1152-1169, 2006.

[16] P. Malliou, A. Gioftsidou, A. Beneka, and G. Godolias, "Measurements and evaluations in low back pain patients". Scandinavian Journal of Medicine and Science in Sports, 219-230, 2006.

[17] A.F. Mannion, F. Balague, F. Pellise, and C. Cedraschi, "Pain measurement in patients with low back pain". Nature Clinical Practice Rheumatology, 3 (11), 610-618, 2007.

[18] M. McCaffery, "Pain: Clinical Manual for Nursing Practice". Mosby: St. Louis, 1989, 2nd edition.

[19] A.H. McGregor, C.J. Dore, I.D. McCarthy, and S.P. Hughes, "Are Subjective Clinical Findings and Objective Clinical Tests Related to the Motion Characteristics of Low Back Pain Subjects?". The Journal of Orthopaedic \& Sports Physical Therapy, 28(6), 370-377, 1998.

[20] V. Mooney, D. Cairns, and J. Robertson, "A system for evaluating and treating chronic back disability". West J. Med. 124, 370-376, 1976.

[21] D.D. Ohnmeiss, "Repeatability of Pain Drawings in a Low Back Pain Population", Spine, 25 (8), 980-988, 2000.

[22] Quesenbery, W. "What Does Usability Mean: Looking Beyond 'Ease of Use"'. Proceedings of the $48^{\text {th }}$ Annual Conference, Society for Technical Communication, 2001.

[23] A.O. Ransford, D. Cairns, and V. Mooney, "The Pain Drawing as an Aid to Psychologic Evaluation of Patients with Low-Back pain", Spine, 1 (2), 127-134, 1976.

[24] K. Samauelsson, H. Larsson, M. Thyberg, and H. Tropp, "Back Pain and Spinal Deformity - Common Among Wheelchair Users with Spinal Cord Injuries". Scandinavian Journal of Occupational Therapy, 3, 2832, 1996.

[25] T. Serif, and G. Ghinea, "Recording of Time-Varying Back-Pain Data: A Wireless Solution". IEEE Transactions on Information Technology in Biomedicine, 9 (3), 447-458, 2005.

[26] M.D. Stinson, S.A. Crawford, and A.P. Porter-Armstrong, "Interface pressure measurements: Visual interpretation of pressure maps with MS clients," Disability and Rehabilitation, 30 (8), 618-624, 2008

[27] M.D. Stinson, A.P. Porter-Armstrong, and P.A. Eakin, "Pressure mapping systems: reliability of pressure map interpretation," Clinical Rehabilitation, 37, 504-511, 2003

[28] K. Takata, and H. Hirotani, "Pain drawing in the evaluation of low back pain". International Orthopaedics, 361-366, 1995.

[29] Y. Tanimoto, H. Takechi, H. Nagahata, and H. Yamamoto, "Pressure Measurement of Cushions for Patients," IEEE, 1245-1250, 1999.

[30] M.L. Vaughn, S.J. Cavill, S.J. Taylor, M.A. Foy, and A.J.B. Fogg, "Using Direct Explanations to Validate a Multi-layer Perceptron Network that Classifies Low Back Pain Patients”, IEEE, 692-699, 1999. 